\title{
31. NANNOFOSSIL AGE DETERMINATIONS, DSDP LEG 16
}

\author{
Stefan Gartner, Jr., Rosenstiel School of Marine and Atmospheric Science, University of Miami, Coral Gables, Florida
}

\section{INTRODUCTION}

The following are age determinations made by means of calcareous nannofossils on selected samples from cores recovered during Leg 16 of the Deep Sea Drilling Project. For the most nart, age determinations were made only for the bottom of every core. The poor nannofossil assemblages recovered almost uniformly throughout this leg make it difficult, and often impossible, to make accurate age (zonal) determinations, so that the relatively broader age assignment that can be made does not require more closely spaced determinations.

No attempt was made to give a comprehensive assemblage list for any of the samples, and generally only key species were sought. In many cases, however, the species listed for a particular sample constitute, for all practical purposes, the entire assemblage. It is not uncommon in these samples to encounter no more than three or four species on a slide. Moreover, the preservation is generally very poor and whole specimens are not very abundant. In some instances the assemblage indicates a much narrower age range than is assigned to it, but zonal assignments are not made for the sake of uniformity. Where key species are listed, the reader will readily recognize them and thereby be able to make his own zonal assignments. Because of the preponderence of inadequately datable samples no general zonation scheme was followed.

It should be clear that the following list represents little more than data. It is obvious, for example, that the age of a particular sample must be middle Oligocene, even if no key species are present, if the samples above and below it are of middle Oligocene age. But even in such a case, the age assignment made is only as restricted as is permitted by the assemblage actually recovered from a sample.

The reason for the poor nannofossil assemblages appears to be solution. The assemblages recovered seem to consist almost entirely of solution-resistant species. Unfortunately many of these same species were relatively long-lived and hence do not permit more accurate age determination.

\section{DSDP 155}

$155-1-1(47-48 \mathrm{~cm})$ :

Reticulofenestra pseudoumbilica, Cyclococcolithina macintyrei, Sphenolithus abies, Coccolithus pelagicus, Discoaster brouweri, cf. Discoaster quinqueramus.

Age: Probably late Miocene.

155-1-3(2-3 cm):

Reticulofenestra pseudoumbilica, Sphenolithus abies, Helicopontosphaera kamptneri, Coccolithus pelagicus, cf. Discoaster quinqueramus.

Age: Probably as above.
155-2-1(3-4 cm):

Sphenolithus abies, Cyclococcolithina leptopora, Coccolithus pelagicus, cf. Discoaster quinqueramus.

Age: Probably as above.

155-3-1(3-4 cm):

Sphenolithus abies, Coccolithus pelagicus, cf. Discoaster quinqueramus, Discoaster brouweri rutellus.

Age: Probably late Miocene.

155-4-1 $(8-9 \mathrm{~cm})$ :

Sphenolithus abies, Reticulofenestra pseudoumbilica, Gephyrocapsa sp.

Age: Neogene, probably a contaminated sample.

155-5-1(0-2 cm):

Coccolithus miopelagicus, Reticulofenestra pseudoumbilica, Sphenolithus abies, Cyclicargolithus floridanus.

Age: Probably middle Miocene.

155-6-2(7-8 cm):

Barren of nannofossils.

155-7-1(38-39 cm):

Coccolithus pelagicus, Cyclicargolithus floridanus, Reticulofenestra umbilica, Sphenolithus abies, Discoaster asymmetricus, Reticulofenestra pseudoumbilica, Discoaster $\mathrm{sp}$.

Age: Probably middle Miocene, contaminated sample.

155-8-1(35-36 cm):

Coccolithus pelagicus, Helicopontosphaera sp., Reticulofenestra pseudoumbilica, cf. Sphenolithus abies.

Age: Probably middle Miocene.

155-8-2(3-4 cm):

Reticulofenestra pseudoumbilica, cf. Sphenolithus abies, Coccolithus pelagicus.

Age: Probably middle Miocene.

\section{DSDP 157}

157-1-1(37-38 cm):

Gephyrocapsa oceanica.

Age: Mid- to late Pleistocene.

157-2-1(6-7 cm):

Gephyrocapsa caribbeanica, Helicopontosphaera kamptneri, Cyclococcolithina leptopora.

Age: Mid- to late Pleistocene.

157-3-1(7-8 cm):

Pseudoemiliania lacunosa, Gephyrocapsa oceanica.

Age: Early to mid-Pleistocene.

157-4-1(54-55 cm):

Gephyrocapsa oceanica, Umbilicosphaera mirabilis, Helicopontosphaera kamptneri, Cyclococcolithina leptopora, Pseudoemiliania lacunosa.

Age: As above. 
157-5-1(8-9 cm):

Gephyrocapsa oceanica, Pseudoemiliania lacunosa.

Age: As above.

157-6-1(6-7 cm):

Pseudoemiliania lacunosa, Cyclococcolithina leptopora.

Age: Probably early Pleistocene.

157-7-1(85-86 cm):

Pseudoemiliania lacunosa, Gephyrocapsa sp., Cyclococcolithina leptopora.

Age: Early Pleistocene.

157-8-1(13-14 cm):

Pseudoemiliania lacunosa, Gephyrocapsa cf. G. caribbeanica, Helicopontosphaera kamptneri, Sphenolithus abies, Gephyrocapsa sp., Gephyrocapsa oceanica.

Age: Probably early Pleistocene, contaminated sample.

$157-9-1(88-89 \mathrm{~cm})$ :

Insufficient material for determination.

$157-10-1(24-25 \mathrm{~cm})$ :

Gephyrocapsa sp., Pseudoemiliania lacunosa.

Age: Early Pleistocene.

157-11-1(3-4 cm):

Pseudoemiliania lacunosa, Cyclococcolithina leptopora, Coccolithus pelagicus.

Age: Probably early Pleistocene.

157-12-1(1-10 cm):

Pseudoemiliania lacunosa, Cyclococcolithina leptopora, Cyclococcolithina mcintyrei, Coccolithus pelagicus, Discoaster sp.

Age: Late Pliocene.

157-13-1(4-5 cm):

Pseudoemiliania lacunosa, Cyclococcolithina leptopora.

Age: Probably late Pliocene.

157-14-1(81-82 cm):

Spenolithus sp., Coccolithus pelagicus.

Preservation inadequate for determination.

157-15-1(45 cm):

Preservation inadequate.

157-16-2(4-5 cm):

Preservation inadequate.

157-17-1(4-5 cm):

Reticulofenestra pseudoumbilica, Sphenolithus abies, Coccolithus pelagicus.

Age: Probably early Pliocene.

$157-18-1(25-26 \mathrm{~cm})$ :

Reticulofenestra pseudoumbilica, Coccolithus pelagicus, Cyclococcolithina leptopora, Helicopontosphaera kamptneri.

Age: Probably early Pliocene.

157-19-1(4-5 cm):

Reticulofenestra pseudoumbilica, Cyclococcolithina leptopora.

Age: Middle Miocene to middle Pliocene.

$157-21-1(4-5 \mathrm{~cm})$ :

Reticulofenestra pseudoumbilica, Coccolithus pelagicus,

Cyclococcolithina leptopora.

Age: As above.
157-22-1(4-5 cm):

Reticulofenestra pseudoumbilica, Cyclococcolithina leptopora.

Age: As above.

157-23-1 $(4-5 \mathrm{~cm})$ :

Reticulofenestra pseudoumbilica, Cyclococcolithina leptorpa, Coccolithus pelagicus.

Age: As above.

157-24-2(4-5 cm):

Reticulofenestra pseudoumbilica, Sphenolithus abies, Cyclococcolithina leptopora.

Age: As above.

$157-25-1(1-10 \mathrm{~cm})$ :

Coccolithus pelagicus, Reticulofenestra pseudoumbilica, Sphenolithus abies, Cyclococcolithina mcintyrei, Cyclococcolithina leptopora Discoaster spp.

Age: As above.

157-26-1 $(4-5 \mathrm{~cm})$ :

Reticulofenestra pseudoumbilica.

Age: Middle Miocene to middle Pliocene.

157-27-1(4-5 cm):

Sphenolithus sp., cf. Reticulofenestra pseudoumbilica, Coccolithus pelagicus, Sphenolithus abies.

Age: Middle Miocene to middle Pliocene.

157-28-3(18-19 cm):

Cyclococcolithina leptopora, cf. Sphenolithus abies, Reticulofenestra pseudoumbilica.

Age: Middle Miocene to middle Pliocene.

157-29-2(10-11 cm):

Insufficient nannofossils.

$157-30-1(14-15 \mathrm{~cm})$ :

Reticulofenestra pseudoumbilica, Sphenolithus sp., Cyclococcolithina leptopora.

Age: Middle Miocene to middle Pliocene.

157-31-1(105-106 cm):

Insufficient nannofossils.

157-32-1(4-5 cm):

Reticulofenestra pseudoumbilica, Cyclococcolithina leptopora.

Age: Middle Miocene to middle Pliocene.

157-33-1(4-5 cm):

Reticulofenestra pseudoumbilica, Cyclococcolithina leotopora, Coccolithus pelagicus, Sphenolithus abies.

Age: Middle Miocene to middle Pliocene.

$157-35-1(4-5 \mathrm{~cm})$ :

Reticulofenestra pseudoumbilica, Cyclococcolithina leptopora, cf. Sphenolithus abies, Coccolithus pelagicus.

Age: As above.

157-36-1(70-71 cm):

Sphenolithus sp., Cyclococcolithina leptopora, Reticulofenestra pseudoumbilica, Sphenolithus abies.

Age: As above.

157-42-1 $(9-10 \mathrm{~cm})$ :

Sphenolithus abies, Coccolithus pelagicus, Reticulofenestra pseudoumbilica.

Age: As above. 


\section{DSDP 157A}

157A-1-2(4-5 cm):

Gephyrocapsa oceanica.

Age: Mid-to late Pleistocene.

157A-1-6(5-6 cm):

Gephyrocapsa oceanica.

Age: As above.

157A-3-3(4-5 cm):

Gephyrocapsa oceanica.

Age: As above.

157A-3-6(8-9 cm):

Gephyrocapsa oceanica, Umbilicosphaera mirabilis,

Pseudoemiliania lacunosa.

Age: Early to mid-Pleistocene.

\section{DSDP 158}

158-1-1(3-4 cm):

Gephyrocapsa oceanica, Emiliania huxleyi.

Age: Late Pleistocene-Holocene.

158-1-3(9-10 cm):

Gephyrocapsa oceanica, Cyclococcolithina leptopora, Gephyrocapsa aperta.

Age: Mid- to late Pleistocene.

158-2-5 $(9-10 \mathrm{~cm})$ :

Gephyrocapsa oceanica.

Age: As above.

158-3-6(9-10 cm):

Gephyrocapsa sp., Helicopontosphaera wallichi, Gephyrocapsa aperta, Pseudoemiliania lacunosa.

Age: Early to mid-Pleistocene.

158-4-4(3-4 cm):

Gephyrocapsa oceanica, Cyclococcolithina leptopora, Pseudoemiliania lacunosa.

Age: As above.

158-5-6(4-5 cm):

Sphenolithus abies, Reticulofenestra pseudoumbilica, $C y$ clococcolithina leptopora, Discoaster sp..

Age: Probably early Pliocene.

158-6-6(3-4 cm):

Discoaster sp., Reticulofenestra pseudoumbilica, Sphenolithus abies, Discoaster pentaradiatus.

Age: Probably as above.

$158-7-6(4-5 \mathrm{~cm})$ :

Reticulofenestra pseudoumbilica, Cyclococcolithina leptopora, Sphenolithus abies.

Age: Probably late Miocene to early Pliocene.

158-8-6 $(4-5 \mathrm{~cm})$ :

Reticulofenestra pseudoumbilica, Sphenolithus abies, Discoaster pentaradiatus, Cyclococcolithina leptorpora, Discoaster spp..

Age: Late Miocene to early Pliocene.

158-9-6 $(9-10 \mathrm{~cm})$ :

Reticulofenestra pseudoumbilica, Discoaster sp., Cyclococcolithina leptopora, Sphenolithus abies, Ceratolithus tricorniculatus, cf. Discoaster brouweri, Coccolithus pelagicus. Age: Late Miocene to early Pliocene.
158-10-6(8-9 cm):

Reticulofenestra pseudoumbilica, Cyclococcolithina leptopora, Sphenolithus abies, Discoaster quinqueramus, Discoaster pentaradiatus, Discoaster variabilis.

Age: Late Miocene.

158-11-6(3-4 cm):

Reticulofenestra pseudoumbilica, Sphenolithus abies, Coccolithus pelagicus, Cyclococcolithina leptopora, Discoaster quinqueramus, Discoaster surculus.

Age: Late Miocene.

158-12-6(4-5 cm):

Reticulofenestra pseudoumbilica, Cyclococcolithina leptopora, Sphenolithus abies, cf. Discoaster quinqueramus. Age: Probably late Miocene.

158-13-6(3-4 cm):

Reticulofenestra pseudoumbilica, Discoaster quinqueramus, cf. Discoaster brouweri.

Age: Late Miocene.

158-14-6(3-4 cm):

Discoaster pentaradiatus, Reticulofenestra pseudoumbilica, Cyclococcolithina leptopora, Discoaster quinqueramus.

Age: Late Miocene.

158-15-6(3-4 cm):

cf. Discoaster neohamatus, Reticulofenestra pseudoumbilica, Coccolithus pelagicus.

Age: Late Miocene.

158-16-6(8-9 cm):

Coccolithus pelagicus, Sphenolithus abies, cf. Discoaster neohamatus, Reticulofenestra pseudoumbilica.

Age: Late Miocene.

158-17-6(4-5 cm):

Reticulofenestra pseudoumbilica, Sphenaster metula, Sphenolithus abies, Coccolithus pelagicus, Cyclococcolithina leptopora.

Age: Probably as above.

158-18-6(4-5 cm):

Coccolithus pelagicus, Sphenolithus abies, Cyclococcolithina leptopora, Reticulofenestra pseudoumbilica.

Age: Probably as above.

158-19-6(8-9 cm):

Reticulofenestra pseudoumbilica, Sphenolithus abies, Discoaster neohamatus.

Age: Late Miocene.

158-20-6(7-8 cm):

Coccolithus pelagicus, Sphenolithus abies, cf. Discoaster hamatus, Discoaster variabilis.

Age: Probably late middle Miocene.

158-21-5(8-9 cm):

Discoaster neohamatus, cf. Discoaster hamatus, Sphenolithus abies, Coccolithus pelagicus.

Age: Probably late middle Miocene.

158-22-6(6-7 cm):

cf. Discoaster hamatus, Reticulofenestra pseudoumbilica, Coccolithus pelagicus.

Age: Probably as above. 
158-23-5(4-5 cm):

Reticulofenestra pseudoumbilica, cf. Discoaster variabilis, Cyclococcolithina leptopora, Sphenolithus abies.

Age: Probably as above.

158-24-3(4-5 cm):

Reticulofenestra pseudoumbilica, Discoaster sp.

Age: Probably as above.

158-25-6(8-9 cm):

Reticulofenestra pseudoumbilica, Discoaster sp., cf. Discoaster quinqueramus.

Age: Late Miocene.

158-26-6(6-7 cm):

Reticulofenestra pseudoumbilica, Discoaster sp., Coccoli-

thus pelagicus, Cyclococcolithina leptopora.

Age: Middle Miocene to middle Pliocene.

158-27-6 $(9-10 \mathrm{~cm})$ :

Reticulofenestra pseudoumbilica, Sphenolithus abies, Cyclococcolithina leptopora.

Age: As above.

158-28-6(4-5 cm):

Reticulofenestra pseudoumbilica, Coccolithus pelagicus.

Age: As above.

158-29-3 $(5-6 \mathrm{~cm})$ :

Reticulofenestra pseudoumbilica, Discoaster sp.

Age: As above.

158-30-6 $(8-9 \mathrm{~cm})$ :

Reticulofenestra pseudoumbilica.

Age: Probably as above.

158-31-1 $(3-4 \mathrm{~cm})$ :

Coccolithus pelagicus, Reticulofenestra pseudoumbilica,

Cyclococcolithina leptopora.

Age: As above.

158-32-4(7-8 cm):

Reticulofenestra pseudoumbilica, Coccolithus pelagicus,

Cyclococcolithina leptopora.

Age: Probably as above.

158-33-2 $(8-9 \mathrm{~cm})$ :

Coccolithus pelagicus, Reticulofenestra pseudoumbilica.

Age: Probably as above.

158-34-4(3-4 cm):

Coccolithus pelagicus, Sphenolithus abies, Reticulofenestra pseudoumbilica.

Age: Probably as above.

\section{DSDP 159}

$159-3-1(4-5 \mathrm{~cm}):$

Discoaster variabilis, Cyclococcolithina leptopora, cf. Discoaster exilis.

Age: Probably middle Miocene.

159-3-6(4-5 cm):

Discoaster $\mathrm{sp}$.

Insufficient nannofossils.

159-4-6(5-6 cm):

Sphenolithus heteromorphus, Cyclicargolithus floridanus,

Discoaster spp.

Age: Early middle Miocene.
159-5-6(9-10 cm):

Coccolithus pelagicus, Cyclicargolithus floridanus, Cyclolithella nitescens, Discoaster spp.

Age: Probably early Miocene.

159-6-4(4-5 cm):

Cyclolithella nitescens, Triquetrorhabdulus carinatus, $C y$ clicargolithus floridanus.

Age: Early Miocene.

$159-7-6(4-5 \mathrm{~cm})$ :

Cyclicargolithus floridanus.

Insufficient nannofossils.

159-8-6(8-9 cm):

Cyclicargolithus floridanus, Discoaster calculosus, Triquetrorhabdulus carinatus.

Age: Early Miocene.

159-9-6 $(12-13 \mathrm{~cm})$ :

Cyclicargolithus floridanus, Coccolithus pelagicus, Sphenolithus abies, cf. Triquetrorhabdulus carinatus.

Age: Early Miocene.

159-10-6(4-5 cm):

Cyclicargolithus floridanus, Coccolithus pelagicus, cf. Triquetrorhabdulus carinatus.

Age: Early Miocene.

159-12-5(7-8 cm):

Cyclicargolithus floridanus, Discoaster calculosus, Triquetrorhabdulus carinatus.

Age: Early Miocene.

159-13-6(14-15 cm):

Cyclicargolithus floridanus, Coccolithus pelagicus, Triquetrorhabdulus carinatus.

Age: Early Miocene.

\section{DSDP 160}

160-4-2(4-5 cm):

Cyclolithella nitescens, cf. Triquetrorhabdulus carinatus, Discoaster sp., Cyclicargolithus floridanus.

Age: Probably early Miocene.

160-4-5(8-9 cm):

Cyclicargolithus floridanus, Coccolithus pelagicus, Triquetrorhabdulus carinatus.

Age: Early Miocene.

160-5-6(9-10 cm):

Cyclicargolithus floridanus, Coccolithus pelagicus, Triquetrorhabdulus carinatus, Discoaster sp.

Age: Early Miocene.

160-6-6(4-5 cm):

Cyclicargolithus floridanus, Coccolithus pelagicus,

Sphenolithus moriformis.

Age: Probably late Oligocene.

160-7-6(8-9 cm):

Cyclicargolithus foridanus, Coccolithus pelagicus, Sphenolithus moriformis, cf. Triquetrorhabdulus milowi. Age: Late Oligocene to early Miocene.

$160-8-5(4-5 \mathrm{~cm})$ :

Sphenolithus distentus, Cyclicargolithus floridanus, Sphenolithus ciperoensis.

Age: Late Oligocene. 
160-9-6(13-14 cm):

Cyclicargolithus floridanus, Sphenolithus distentus, aff. Sphenolithus ciperoensis.

Age: Probably late Oligocene.

160-10-6(6-7 cm):

Cyclicargolithus floridanus, Coccolithus pelagicus, Sphenolithus moriformis, Sphenolithus distentus.

Age: Probably middle Oligocene.

160-11-6(9-10 cm):

Sphenolithus moriformis, Coccolithus pelagicus, Cyclicargolithus floridanus, Sphenolithus predistentus.

Age: Middle Oligocene.

$160-12-4(4-5 \mathrm{~cm})$ :

Cyclicargolithus foridanus, Sphenolithus moriformis, Coccolithus pelagicus, Reticulofenestra scissura, Sphenolithus predistentus.

Age: Middle Oligocene.

160-12-6(9-10 cm):

Cyclicargolithus floridanus, Sphenolithus moriformis, Reticulofenestra scissura, Coccolithus pelagicus, Sphenolithus distentus.

Age: Middle Oligocene.

\section{DSDP 161}

$161-1-1(4-5 \mathrm{~cm})$ :

Barren of nannofossils.

161-1-6(8-9 cm):

Cyclicargolithus floridanus, Discoaster sp.

Age: Early Neogene.

$161-2-6(4-5 \mathrm{~cm})$ :

cf. Triquetrorhabdulus carinatus, Cyclicargolithus floridanus, Discoaster sp.

Age: Early Miocene.

161-3-5(11-12 cm):

Cyclicargolithus floridanus.

Insufficient nannofossils.

161-4-4(8-9 cm):

Coccolithus pelagicus, Discoaster sp., Cyclicargolithus floridanus.

Insufficient nannofossils.

$161-5-6(4-5 \mathrm{~cm})$ :

Cyclicargolithus floridanus, Sphenolithus moriformis, Discoaster sp.

Age: Early Neogene. Insufficient nannofossils.

161-6-6(4-5 cm):

Cyclicargolithus floridanus, Sphenolithus moriformis, Discoaster sp.

Age: Probably early Neogene. Insufficient nannofossils.

161-7-5 $(9-10 \mathrm{~cm})$ :

Cyclicargolithus floridanus, Coccolithus pelagicus,

Sphenolithus moriformis, Discoaster sp.

Age: Early Neogene. Insufficient nannofossils.

161-9-6(4-5 cm):

Sphenolithus moriformis, Cyclicargolithus floridanus, Sphenolithus distentus.

Age: Middle Oligocene.
161-10-6(4-5 cm):

Cyclicargolithus floridanus, Sphenolithus moriformis, Sphenolithus distentus.

Age: Middle Oligocene.

161-11-6(8-9 cm):

Cyclicargolithus floridanus, Coccolithus pelagicus, Sphenolithus moriformis.

Age: Probably as above. Insufficient nannofossils.

$161-12-6(4-5 \mathrm{~cm})$ :

Coccolithus pelagicus, Cyclicargolithus floridanus,

Sphenolithus moriformis.

Age: Probably as above. Insufficient nannofossils.

161-13-6(4-5 cm):

Sphenolithus moriformis, Cyclicargolithus foridanus, Coccolithus pelagicus, Reticulofenestra scissura.

Age: Probably as above. Insufficient nannofossils.

\section{DSDP 161A}

161A-1-2 (8-9 cm):

Cyclicargolithus floridanus, Coccolithus pelagicus, Sphenolithus moriformis, Sphenolithus distentus.

Age: Middle Oligocene.

161A-1-6(11-12 cm):

Cyclicargolithus floridanus, Discoaster sp., Sphenolithus moriformis, Coccolithus pelagicus, Sphenolithus distentus. Age: Middle Oligocene.

161A-2-6(8-9 cm):

Cyclicargolithus floridanus, Sphenolithus moriformis.

Age: Probably as above.

161A-3-6(4-5 cm):

Reticulofenestra scissura, Sphenolithus moriformis, Coccolithus pelagicus, Cyclicargolithus floridanus, cf. Sphenolithus distentus.

Age: Probably middle Oligocene.

161A-4-6 $(9-10 \mathrm{~cm})$ :

Sphenolithus distentus.

Age: Middle Oligocene.

161A-5-6(7-8 cm):

Coccolithus pelagicus, Cyclicargolithus floridanus, cf.

Sphenolithus distentus.

Age: Middle Oligocene.

161A-6-6(9-10 cm):

Cyclicargolithus floridanus, Sphenolithus moriformis.

Age: Probably as above. Insufficient nannofossils.

161A-7-6(8-9 cm):

Coccolithus pelagicus, Cyclicargolithus floridanus,

Reticulofenestra scissura, Sphenolithus distentus.

Age: Middle Oligocene.

161A-8-3(8-9 cm):

Cyclicargolithus floridanus, Coccolithus pelagicus, Reticulofenestra scissura, Sphenolithus predistentus.

Age: Early to middle Oligocene.

161A-9-5 $(4-5 \mathrm{~cm})$ :

Reticulofenestra scissura, Cyclicargolithus floridanus, Coccolithus pelagicus, Cyclolithella nitescens, Cyclococcolithina formosa, Reticulofenestra umbilica.

Age: Early Oligocene. 
161A-10-6(8-9 cm):

Insufficient nannofossils.

161A-11-5 (8-9 cm):

cf. Discoaster saipanensis.

Age:Probably late Eocene.

161A-12-1 $(8-9 \mathrm{~cm})$ :

Reticulofenestra scissura, cf. Discoaster siapanensis, cf. Discoaster barbadiensis.

Age: Late Eocene.

161A-14-2(4-5 cm):

Insufficient nannofossils.

\section{DSDP 162}

$162-1-1(4-5 \mathrm{~cm})$ :

Barren of calcareous nannofossils.

162-1-6(12-13 cm):

Sphenolithus moriformis, Cyclicargolithus foridanus,

Sphenolithus distentus.

Age: Middle Oligocene.

162-2-6(8-9 cm):

Reticulofenestra scissura, Sphenolithus moriformis, Discoaster sp., Cyclicargolithus floridanus.

Age: Probably early Oligocene. Nannofossil preservation very poor.

$162-3-6(4-5 \mathrm{~cm})$ :

Reticulofenestra scissura, Cyclococcolithina formosa,

Sphenolithus moriformis.

Age: Early Oligocene.

$162-4-6(4-5 \mathrm{~cm})$ :

Barren of calcareous nannofossils.

162-5-6(8-9 cm):

Barren of calcareous nannofossils.

$162-6-4(4-5 \mathrm{~cm})$ :

Barren of calcareous nannofossils.

$162-7-6(4-5 \mathrm{~cm})$ :

Barren of calcareous nannofossils.

162-8-6(15-14 cm):

Discoaster barbadiensis, Reticulofenestra umbilica.

Age: Probably late middle Eocene.

162-9-6 $(14-15 \mathrm{~cm})$ :

cf. Discoaster barbadiensis, Cyclococcolithina formosa, cf. Reticulofenestra samodurovi.

Age: Middle Eocene.

162-10-6(4-5 cm):

Discoaster sp., cf. Discoaster barbadiensis.

Insufficient nannofossils.

162-11-6(4-5 cm):

Discoaster barbadiensis.

Age: Probably middle Eocene.

$162-12-6(4-5 \mathrm{~cm})$ :

cf. Discoaster barbadiensis, cf. Reticulofenestra samodurovi, Cyclococcolithina formosa.

Age: Probably middle Eocene.
162-13-6(9-10 cm):

cf.Discoaster barbadiensis, Coccolithus pelagicus, cf. $\mathrm{Cy}$ clococcolithina formosa.

Age: Probably middle Eocene.

162-14-6(4-5 cm):

Discoaster barbadiensis.

Age: Probably middle Eocene.

162-15-6(4-5 cm):

Cyclococcolithina formosa.

Age: Probably middle Eocene. Insufficient nannofossils.

$162-16-2(6-7 \mathrm{~cm})$ :

Barren of calcareous nannofossils.

$162-17-3(4-5 \mathrm{~cm})$ :

Cyclococcolithina gammation, Sphenolithus spiniger, $\mathrm{Cy}$ clococcolithina formosa.

Age: Early to early-middle Eocene.

\section{DSDP 163}

$163-15-1(34-35 \mathrm{~cm}):$

Watznaueria barnesae, Cribrosphaerella ehrenbergi, Micula sp. Micula decussata.

Age: Late Cretaceous. Preservation very poor.

163-15-6(108-109 cm):

Watznaueria barnesae, Cribrosphaerella ehrenbergi, Prediscosphaera cretacea, Micula decussata, Arkhangelskiella sp., Cylindralithus sp.

Age: Late Cretaceous.

$163-16-6(4-5 \mathrm{~cm})$ :

Watznaueria barnesae, Arkhangelskiella sp., Prediscosphaera cretacea, Cretarhabdus sp., Cribrosphaerella ehrenbergi, Cylindralithus sp., Cylindralithus gallicus.

Age: Late Cretaceous, probably Maestrichtian.

163-17-6(8-9 cm):

Watznaueria barnesae, Tetralithus nitidus nitidus, Cribrosphaerella ehrenbergi, Prediscosphaera cretacea, Cylindralithus sp., Cretarhabdus sp.

Age: Late Cretaceous, probably early Maestrichtian.

163-18-6(6-7 cm):

Watznaueria barnesae, Cretarhabdus sp., Cribrosphaerella ehrenbergi.

Age: Late Cretaceous.

163-19-6(4-5 cm):

Prediscosphaera cretacea, Cretarhabdus sp., Watznaueria barnesae, Tetralithus nitidus nitidus, Tetralithus nitidus trifidus, Cylindralithus sp.

Age: Late Cretaceous, Tetralithus nitidus trifidus Zone.

163-20-6(4-5 cm):

Watznaueria barnesae, Prediscosphaera cretacea, Tetralithus nitidus nitidus, Tetralithus nitidus trifidus.

Age: Late Cretaceous, Tetralithus nitidus trifidus Zone.

163-21-6(4-5 cm):

Watznaueria barnesae.

Insufficient nannofossils. 
$163-22-6(4-5 \mathrm{~cm})$ :

Watznaueria barnesae, Prediscosphaera cretacea, Micula decussata Cribrosphaerella ehrenbergi, Tetralithus nitidus nitidus.

Age: Late Cretaceous.

163-23-6(8-9 cm):

Watznaueria barnesae, Micula decussata, Prediscosphaera cretacea.

Age: Late Cretaceous.

$163-26-2(4-5 \mathrm{~cm})$ :

Watznaueria barnesae, Kamptnerius sp., Prediscosphaera cretacea, Micula decussata, Cretarhabdus sp., Eiffellithus turriseiffeli, cf. Cylindralithus serratus, Tetralithus aculeus.

Age: Late Cretaceous, probably late Campanian.

$163-27-1(26-27 \mathrm{~cm})$ :

Watznaueria barnesae, Eiffellithus turriseiffeli, Prediscosphaera cretacea, Tetralithus aculeus, Micula decussata. Age: Probably as above.

\section{DSDP 163A}

163A-1-2(4-5 cm):

Barren of calcareous fossils.

$163 \mathrm{~A}-1-4(10-11 \mathrm{~cm})$ :

Barren of calcareous fossils. 